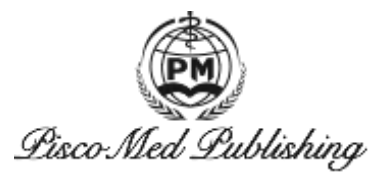

Original Research Article

\title{
On the Application of Dynamic Logos in Brand Image Design
}

\section{Yunxi Wang*}

Sichuan University of Science \& Engineering, Zigong 643000, Sichuan, China. E-mail: racy562526472@163.com

Abstract: Market competition is increasingly fierce in the new era. Enterprises must focus on establishing their good brand images in order to occupy a favorable position in the competition. The effective application of dynamic logos in brand image design can make logos develop towards three-dimensional or even multi-dimensional space, enriching and diversifying brand logos through dynamic graphics, personalized voice, rich colors, etc.

Keywords: Dynamic Logos; Brand Image Design; Application

\section{Introduction}

Logo is an important form in commodity transmission progress. In the process of brand development, logo is its core content, which can not only fully show the image of the brand, but also show the value of the brand. With the development of modern science and technology, modern information technology can give full play to its advantages in brand image design, which provides a broad developing space for the design and enriches and diversifies brand design, promotes the dynamic development and constant application of brand image design.

\section{Main causes of dynamic logos}

\subsection{Development of modern information technology}

The development of modern information technology provides a broader development space for logo design and also provides a broader media platform for the development of dynamic logo design ${ }^{[4]}$. Digital media art embodies new thinking, new technology and new aesthetics in the information age. Dynamic logos are widely accepted and understood by people depending on its characteristics of popularization, network communication and interaction. Under the constant influence of new technologies and new media, the types of dynamic logos will be richer and more diversified. The logos can mobilize people's multiple senses, thus meeting more people's psychological needs.

\subsection{Development of humanized design}

With the development of economy, the competition in the society is becoming increasingly fierce. People are more eager for emotional communication and ideological communication than ever before, which requires design to meet people's spiritual and emotional needs. On the basis of practical functions, logo design must meet people's aesthetic and spiritual needs too. In the process of information dissemination, logo is an important visual element that can express people's emotions. From this perspective, the development of logo design needs to be humanized. Commodity circulation has become faster, and logos have become indispensable parts of commodities. With the diversification of peoples' needs and the continuous improvement of the aesthetic ability, logo design should pay more attention to the

Copyright (C) 2020 Yunxi Wang

doi: $10.18282 / 1-$ e.v9i1.868

This is an open-access article distributed under the terms of the Creative Commons Attribution Non-Commercial License

(http://creativecommons.org/licenses/by-nc/4.0/), which permits unrestricted non-commercial use, distribution, and reproduction in any medium,

provided the original work is properly cited. 
needs of humanized design in terms of function. The design forms should be diversified and novel. Dynamic logos can create more changeable, flexible and colorful sign forms ${ }^{[1]}$ which can better meet individual needs of people. Therefore, the development of humanized design has promoted the development of dynamic logos ${ }^{[9]}$.

\section{Performance of dynamic logos in brand image design}

\subsection{Expression forms in graphics}

Dynamic logos can better present design functions in the process of brand image design. It is more common to show dynamic design effect through static expression. Such dynamic logo is of rhythm and can attract people's attention. The application of most dynamic logo in brand image design has realized the expression of being both static and dynamic. It's seems that one can easily make this kind of expression. However, in fact, it has very high requirements for designers. Designers need to have professional design experience and master relatively high design skills to bring such rich and powerful visual enjoyment to people. In three-dimensional graphics, brand logo has a more dynamic feeling through the application of three-dimensional animation with the help of science and technology, which makes people feel optical illusions ${ }^{[2]}$.

\subsection{Expression forms in sounds}

At present, the design of brand logo has gradually changed to three-dimensional space, and meanwhile, it is also developing towards multi-dimensional space ${ }^{[5]}$. Therefore, the design of brand image is constantly becoming diversified and dynamic. In the past, enterprises promoted brand image through graphic and static brand designs. Nowadays, with the wide application of dynamic logos, simply two-dimensional visual experience can not meet human's satisfactions. People need multi-sensory enjoyment, and have requirements on sound and other aspects. For example, one will receive others' information when he/she logs in to QQ and there will be an instant message QQ with winking the avatar of the contact person, which realizes the effective application of images. There will be drops of sound (like cough) when someone contacts you during your online period. The sound will be different from other people when it comes to someone you concerns about. The effective application of sounds has become a characteristic of QQ, which reflects the important application of sound in dynamic logos and also indicates the important role of effective application of dynamic logos in corporate brand image.

\subsection{Expression forms in color}

The effective application of color is one of the most basic and important part in brand design. Color can bring a very intuitive feeling to people. Through the observation of color, people can understand the connotation conveyed by the logo and can easily distinguish the brands of different enterprises ${ }^{[6]}$. For example, Coca-Cola is red and Pepsi Cola is blue to many consumers. Color is an important factor for people to distinguish some brands. In the process of dynamic logo design for brand image, designers can make static colors into dynamic ones, which enables colors to change continuously, thus making visual images colorful. Colors change with images, which makes a logo eye-catching. Traffic lights are the most common dynamic color presentation. During driving, the driver judges whether they should stop and wait, or drive based on the traffic lights. Designers can accurately convey some symbolic information through dynamic color expressions, bringing convenience to people's lives.

\section{Application characteristics of dynamic logo in brand image design}

\subsection{To improve quality}

In the process of designing the brand image logo for an enterprise, designers need to follow up the principle of simplicity and clarity, which can better publicize the image of the enterprise, and is conducive to making the brand connotation of the enterprise clearer. Besides, designers should also integrate the factors of enterprise culture and concept, and integrate the dynamic design concept into the brand image design of the enterprise, so that the concept 
can be better expressed in a simply and clearly way. The logo can be more textured, and the brand awareness can be more vividly conveyed in this method ${ }^{[3]}$. For example, the original logo of some enterprises is flat and static, and the concept and idea of the enterprise can only be conveyed through vision. With the development of dynamic logo, some of these enterprises begin to realize the shortage of their brand image. Through the application of dynamic brand logo, logos of these enterprises become vivid and dynamic, which can better convey the idea and culture of the enterprise, thus improving the brand quality of the enterprise.

\subsection{Limitation of media}

With the rapid development of modern information technology, the Internet has gradually become a part of people's life, providing a broad space for the development of Internet companies. Some Internet companies have designed more dynamic logos to meet the continuous improvement of people's needs. The application of these dynamic signs not only catches people's eyes, but also leads a trend. Some of these companies adopt the familiar elements in China's traditional culture to design dynamic logos, which has received widespread attention ${ }^{[7]}$. However, they are also limited by the development of modern information technology. Such dynamic signs are mainly electronic media. Once there is no electronic media, dynamic logos will lose attention and become less attracting. To a certain extent, the development and transmission of dynamic logos are limited by media.

\section{Application value of dynamic logo in brand image design}

\subsection{To avoid visual fatigue and get more extensive attention}

It will make people feel visual fatigue to pass information to people only through the way of two-dimensional graphics. Today, the application of dynamic logos in brand image design can attract people's attention, which enables information to be transmitted more effectively, not only making it easier for people to accept the brand image, but also enabling people to better understand its connotation.

\subsection{To enhance emotion of brand images}

With the development of economy and society, people's thoughts have been continuously improving. Currently, the design of brand image attaches great importance to the concept of people-oriented, and has strengthened the application of humanism to the design of brand image of enterprises. While brand logo plays an active role in communication, it should also pay attention to more humanistic care for consumers. This shows that the essence of design is to better meet people's material and spiritual needs. The application of dynamic logos in brand image designs of an enterprise can present the connotation of the enterprise more vividly. Dynamic graphics can connect the enterprise with consumers, increase their interaction and enhance their interest better, which can not only fully present the brand charm of the enterprise, but also cause emotional resonance with consumers.

\subsection{To show individual characteristic}

Dynamic logo has its novel and unique multi-dimensional design features by showing through modern digital technology and new media, which gives people an unusual and unique visual experience ${ }^{[8]}$. It can avoid the homogenization of static logo modeling. This is a brand-new effect achieved by dynamic logo compared with static logo.

\section{Conclusion}

Under the new circumstances, traditional brand image design methods are no longer adapted to the development trend of the times. Designers need to strengthen the continuous optimization and innovation of dynamic logo design methods, and make full use of graphics, colors and sounds, etc. In brand image design, designers also need to make logos more vivid and diversified to promote the forward development of brand logos with the deeper application of dynamic logos. 


\section{References}

1. Jing D, Qu Y. Application of dynamic logo design in brand image (in Chinese). Shanhaijing 2019; (5): 21.

2. Guo Y. Basic principles of logo design based on brand value (in Chinese). Western Leather 2018; 40(20): 23.

3. Gao T. Dynamic diversification of brand image design research (in Chinese). Grand View (Tokyo Literature) 2019; (9): $3-4$.

4. Duan H. On the new trend of sign development in new media age. Da Guan 2017; (7): 64.

5. Tian Z, Li Y. Application of dynamic sign in brand image design. Literature Life (Next Trimonthly Publication) 2012; 11: 177-178.

6. Wang X, Zhan Y. The application of color in brand design. The artists 2019; 7.

7. Liu L, Xu M. Analysis of dynamic sign design form in new media environment. Home Drama 2016; (4): 173-174.

8. Shi S. Personalized representation of logo design (in Chinese). Economic \& Trade Update 2008; 6(S5): 228-229.

9. Zhou Z. On the dynamic development of sign. News Dissemination 2018; 12: 7-11. 